\title{
Slit2 inhibits glioma cell invasion in the brain by suppression of Cdc42 activity
}

\author{
Jia-Jean Yiin, Bo Hu, Michael J. Jarzynka, Haizhong Feng, Kui-Wei Liu, Jane Y. Wu, \\ Hsin-I Ma, and Shi-Yuan Cheng \\ University of Pittsburgh Cancer Institute (J.-J.Y., B.H., M.J.J., H.F., K.W.L., S.-Y.C.), Department of Pathology \\ (J.-J.Y., M.J.J., H.F., K.W.L., S.-Y.C.), and Department of Medicine (B.H.), University of Pittsburgh, Pittsburgh, \\ PA, USA; Department of Neurological Surgery, Tri-Service General Hospital and Graduate Institute of Medical \\ Sciences, National Defense Medical Center, Taipei, Taiwan (J.-J.Y., H.-I.M.); Veterans Hospital, Taoyuan, \\ Taiwan (J.-J.Y.); Department of Neurology, Northwestern University Feinberg Medical School, Robert H. Lurie \\ Comprehensive Cancer Center, Center for Genetic Medicine, Chicago, IL, USA (J.Y.W.)
}

Acquisition of insidious invasiveness by malignant glioma cells involves multiple genetic alterations in signaling pathways. Slit2, a chemorepulsive factor, controls cell migration of neuronal and glial cells during development and inhibits chemotaxic migration of various types of cells in vitro. However, the role of Slit 2 in vitro remains controversial, and the biological significance of Slit 2 expression in cancer cell invasion in vivo has not yet been determined. In the present study, we characterized the effects of Slit2 expression on the migration and invasion of invasive glioma cells in vitro and in vivo. By reverse transcriptase polymerase chain reaction (PCR) analyses, Slit 2 was found to be expressed at lower levels in primary glioma specimens and invasive glioma cells compared with normal human brain cells and astrocytes. Ectopic expression of Slit 2 or treatment with recombinant Slit 2 on glioma cells attenuates cell migration and invasion through inhibition of $\mathrm{Cdc} 42$ activity in vitro. Cellular depletion of Robo1, a cognate receptor for Slit2, prevented Slit2 inhibition of Cdc42

Received July 14, 2008; accepted January 24, 2009.

Address correspondence to Shi-Yuan Cheng, Department of Pathology, University of Pittsburgh Cancer Institute, Research Pavilion at the Hillman Cancer Center, 2.26f, 5117 Centre Ave., Pittsburgh, PA 15213, USA (chengs@upmc.edu), or Bo Hu, Department of Medicine, University of Pittsburgh Cancer Institute, Research Pavilion at the Hillman Cancer Center, 2.19, 5117 Centre Ave., Pittsburgh, PA 15213, USA (hub@upmc.edu). activity and glioma cell migration. In vivo, expression of Slit 2 by invasive SNB19 glioma cells markedly inhibited glioma cell infiltration into the brain of mice. Moreover, impediment of glioma cell invasion by Slit 2 did not affect the expression of $\mathrm{N}$-cadherin and $\beta$-catenin in glioma cells. These results provide the first evidence demonstrating that Slit2-Robo1 inhibits glioma invasion through attenuating $\mathrm{Cdc} 42$ activity in vitro and in the brain. Understanding the mechanisms of Slit2-Robo1 inhibition of glioma cell invasion will foster new treatments for malignant gliomas. Neuro-Oncology 11, 779-789, 2009 (Posted to Neuro-Oncology [serial online], Doc. D0800186, March 31, 2009. URL http://neuro-oncology .dukejournals.org; DOI: 10.1215/15228517-2008-017)

Keywords: Cdc42, glioma, invasion, Slit2

$\mathrm{M}$ alignant gliomas are diffuse, highly invasive, and often multifocal tumors that have a dismal prognosis with a median survival of approximately 16 months. ${ }^{1}$ One of the major obstacles to effective treatment of gliomas is the intrinsic ability of single tumor cell infiltration that extends tendrils of the tumor several centimeters away from the main tumor mass, resulting in incomplete surgical removal and contributing to the high frequency of tumor recurrence. ${ }^{2}$ It has been established that acquisition of invasive behaviors by malignant glioma cells involves multiple and coordinated cellular programs, including those necessary for cell migration and invasion, rendering the tumor cells 
highly resistant to conventional treatments. ${ }^{3}$ Although accumulating evidence demonstrates that various stimuli promote glioma cell infiltration into brain parenchyma, the mechanisms underlying the dysregulation of cell motility during tumor invasion by the insidious glioma cells remain largely unknown.

Slit proteins (Slit1-Slit3) are secreted extracellular matrix (ECM)-associated glycoproteins and ligands for the repulsive guidance receptor Roundabout (Robo1Robo4) family. ${ }^{4}$ In the central nervous system (CNS), the Slit-Robo interactions play an important role in axon guidance. ${ }^{5}$ The Slit proteins control the migration of neurons ${ }^{6}$ and neural crest cells ${ }^{7}$ by enhancing the interaction between novel Slit-regulated Rho GTPaseactivating proteins (srGAPs) and the Robo receptor, thereby inactivating Cdc42, a small Rho GTPase ${ }^{8}$ that is critical in cell motility. ${ }^{9}$ Outside the CNS, Slit2 inhibits in vitro migration of various types of mammalian cells such as leukocytes, ${ }^{10}$ dendritic cells, ${ }^{11}$ and vascular smooth muscle cells. ${ }^{12}$ In human cancers, the Slit2 gene is frequently inactivated by hypermethylation in its promoter region or allele loss in lung, breast, and colorectal cancers and malignant gliomas, ${ }^{13-15}$ suggesting a tumorsuppressive role for Slit2. In breast cancer and medulloblastoma cells, Slit2 inhibits cell migration and invasion in vitro. ${ }^{16,17}$ However, whether Slit 2 inhibits cell migration and invasion of human cancers in vivo has not been demonstrated.

In this study, we determined whether Slit2 inhibits the migration and invasion of glioma in vitro and in the murine brain. We report here that Slit 2 expression by invasive glioma cells impeded cell migration and invasion in vitro. When invasive glioma cells stably expressing Slit 2 were implanted into the brain of mice, glioma cell infiltration into the brain parenchyma was markedly attenuated. The effects of Slit 2 impairment on migration and invasion of glioma cells were mediated by inhibiting Cdc42 activity in migrating cells. Our results also show that Slit2 inhibits human glioma cell invasion in vivo through a distinct mechanism of suppressing Cdc42 activity without affecting the expression of $\mathrm{N}$-cadherin and $\beta$-catenin proteins.

\section{Materials and Methods}

\section{Cell Lines, Antibodies, and Reagents}

We obtained U373MG glioma cells from American Type Culture Collection (Manassas, VA, USA) and cultured them as previously described. ${ }^{18}$ SNB19 glioma cells were a gift from Dr. Y.-H. Zhou, University of California, Irvine. Normal human astrocytes (NHAs) were from Lonza (Allendale, NJ, USA). The following antibodies were used in our studies: goat anti- $\beta$-actin (I-19, SC-1616) and mouse anti-c-Myc (9E10, SC-40 (Santa Cruz Biotechnology, Santa Cruz, CA, USA); anti- $\beta$-catenin (610153; BD Biosciences, San Diego, CA, USA); anti-N-cadherin (3B9, 33-3900; Invitrogen Life Science Zymed, Carlsbad, CA, USA); antiphosphotyrosine (4G10, 05-321, Upstate Biotechnology, Lake
Placid, NY, USA); and mouse anti-N-Cadherin (GC-4, C3865; Sigma Chemicals, St. Louis, MO, USA). Protein $\mathrm{G}$ agarose beads were from Invitrogen, Inc. (Carlsbad, CA, USA). The secondary antibodies were from Dako (Carpinteria, CA, USA) or Molecular Probes (Eugene, OR, USA). Cell culture media and other reagents were from Hyclone (Salt Lake City, UT, USA), Sigma, and Fisher Scientific (Hanover Park, IL, USA).

\section{RNA Isolation and Reverse Transcriptase PCR}

Total RNA was isolated from snap-frozen normal human brain (NB), glioma specimens (from Dr. R. Nishikawa, Saitama, Japan), NHA, and various glioma cell lines using Trizol reagent. Total RNA (5 $\mu \mathrm{g})$ was reverse-transcribed with a superscript reverse transcriptase (Invitrogen Life Science, Carlsbad, CA, USA) using oligo(dT) and random hexanucleotide primers. Polymerase chain reaction (PCR) primers were as follows: human Robo1, sense: 5'-GCA TCG CTG GAA GTA GCC ATA C-3', antisense: 5'-GTG TAT GAA CCC ATG TCA CCA GC-3'; human Slit2, sense: 5'-GGT GTC CTC TGT GAT GAA GAG-3', antisense: 5'-GTG TTT AGG AGA CAC ACC TCG-3'; and $\beta$-actin, sense: 5'-TCC CCC AAC TTG AGA TGT ATG AAG-3', antisense: 5'-AAC TGG TCT CAA GTC AGT GTA CAG G-3'. ${ }^{17}$ The PCR reactions were performed by using $300 \mathrm{nM}$ of each primer under the following conditions: $3 \mathrm{~min}$ at $95^{\circ} \mathrm{C}$ for one cycle followed by 35 cycles of 1 min at $94^{\circ} \mathrm{C}, 1 \mathrm{~min}$ at $58^{\circ} \mathrm{C}$, and $2 \mathrm{~min}$ at $72^{\circ} \mathrm{C}$. Each reverse transcriptase (RT)-PCR product $(6 \mu \mathrm{l})$ was analyzed by electrophoresis on a $2 \%$ agarose gel (SeaKem LE, catalog no. BMA 50000. Images of the gels stained with ethidium bromide were captured with a digital video image analyzer (BioVision, Exton, PA, USA).

\section{Expression of Human Slit2 in Glioma Cells}

SNB19 and U373MG cells were seeded at 70\% density into $100 \mathrm{~mm}$ dishes. On the following day, the cells were transfected with $2 \mu \mathrm{g}$ hSL2myc cDNA containing a 6-cMyc $\operatorname{tag}^{17}$ using Effectene transfection reagent (Qiagen, Inc., Valencia, CA, USA). After $24 \mathrm{~h}$, the cells were selected in medium containing $500 \mu \mathrm{g} / \mathrm{ml}$ Geneticin (G-418 sulfate; Invitrogen) for 3-4 weeks. Geneticinresistant cell clones that showed no alteration of their growth and survival were characterized for ectopic Slit2 expression by immunoblot (IB) analyses.

\section{Intracranial Xenograft and Histological Analyses}

The intracranial glioma xenograft model was performed as previously described. ${ }^{19}$ Briefly, $1 \times 10^{6}$ of SNB19 parental and Slit2-expressing cell clones in $5 \mu \mathrm{l}$ phosphate-buffered saline (PBS) were stereotactically implanted into the brain of 8-week-old female mice (Ncr, athymic nu/nu; Taconic Farms, Germantown, PA, USA). At 7, 14, and 20 days postimplantation, mice were euthanized and their brains were removed, embedded, and stored at $-80^{\circ} \mathrm{C}$. Brain tissues were sectioned on a 
cryostat in $8-\mu \mathrm{m}$ thickness and stained with hematoxylin and eosin.

\section{Cell Viability and Wound Healing Assays}

Cell viability assays were performed as previously described. ${ }^{20}$ Four thousand various glioma cells were seeded per well in a 96-well plate. Twenty-four hours later, the cells were serum-starved for $24 \mathrm{~h}$ before addition of fresh media containing 1\% fetal bovine serum (FBS) and $0.05 \%$ bovine serum albumin (BSA). At 24 and $48 \mathrm{~h}$, cell viability was measured by using the WST-1 reagent (Roche Applied Sciences, Indianapolis, IN, USA) following the manufacturer's recommendations. Wound healing experiments were performed as previously described. ${ }^{21}$ Briefly, glioma parental or Slit2expressing cell clones were seeded on six-well plates with Dulbecco's modified Eagle's medium (DMEM) containing 10\% FBS and grown to confluence. Cells were scratched with a sterile $200-\mu$ l pipette tip to create an artificial wound. At 0,24 , and $48 \mathrm{~h}$ after wounding, phase-contrast images of the wound-healing process were photographed digitally using an inverted microscope (model IX50; Olympus America, Inc., Center Valley, PA, USA).

\section{In Vitro Migration and Invasion Assays}

In vitro migration and invasion assays were performed as previously described. ${ }^{19}$ Briefly, $5 \times 10^{5} \mathrm{SNB} 19$ cells $/ \mathrm{ml}$ and $1 \times 10^{6} \mathrm{U} 373 \mathrm{MG}$ cells/ml in $50 \mu \mathrm{l}$ DMEM containing $0.5 \% \mathrm{FBS}$ and $0.05 \% \mathrm{BSA}$ were separately placed into the top compartments of a 48-well Boyden chamber (Neuro Probe, Inc., Gaithersburg, MD, USA). The cells were allowed to migrate through a polycarbonate membrane ( $8 \mu \mathrm{m}$ pores; Neuro Probe, Inc.) precoated with gelatin $(250 \mu \mathrm{g} / \mathrm{ml})$ or invade through a membrane precoated with Growth Factor Reduced Matrigel (0.78 $\mathrm{mg} / \mathrm{ml}$; BD Biosciences, Boston, MA, USA) for 8 or 16 $\mathrm{h}$ at $5 \% \mathrm{CO}_{2}$ and $37^{\circ} \mathrm{C}$. Afterward, the membrane was fixed and stained, nonmigrating and noninvading cells were removed, and the remaining cells were counted with a total magnification $\times 100$ under an Olympus BX51 microscope.

\section{Small Interfering RNAs and Transfections}

A pool of four small interfering RNAs (siRNAs) for Robo1 (siGENOME SMARTpool, M-011381-00) was synthesized by Dharmacon (Lafayette, CO, USA). Transfection of glioma cells with the aforementioned siRNA was performed using Lipofectamine 2000 (Invitrogen Life Science) as previously described. ${ }^{22}$ Mock transfection was done in parallel using the Stealth RNAi Negative Control Med GC (Invitrogen Life Science). After $24 \mathrm{~h}$, the siRNA/lipid complexes were removed, and the cells were maintained in complete medium for an additional $48 \mathrm{~h}$. The inhibition of Robo1 expression was determined by RT-PCR analyses.

\section{Immunoblot, Immunoprecipitation, and Immunodepletion Analyses}

The IB, immunoprecipitation (IP), and immunodepletion analyses of various cells were performed as previously described. ${ }^{19,23-25}$ Antibodies used in analyses were, for IB, anti-c-Myc (SC-40, 1:1,000), anti-N-cadherin (3B9, $1: 500)$, anti- $\beta$-catenin $(610153,1: 500)$, antiphosphotyrosine (4G10, 1:500), and $\beta$-actin (SC-1616, 1:500); for IP, $5 \mu \mathrm{g}$ of anti-N-cadherin (3B9) or $\beta$-catenin antibody; and for immunodepletion, an anti-c-Myc antibody (SC-40, $20 \mu \mathrm{g} / \mathrm{ml})$.

\section{Cdc42 Activation Assay}

Guanosine-5'-triphosphate (GTP) loading of Cdc42 was measured using the Cdc42 Activation Assay Kit (Upstate Technology), according to manufacturer's instructions. Briefly, cells were lysed in ice-cold magnesium lysis buffer and cleared with glutathione-agarose beads. Cell extracts were then incubated with PAK-1 PBD agarose beads included in the kit, pelleted, and washed. The beads were resuspended in sample buffer and separated by $10 \%$ polyacrylamide gel electrophoresis. GTP-bound Cdc42 was detected using an anti-Cdc42 antibody.

\section{Statistical Analysis}

A one-way analysis of variance with Newman-Keuls post test or a paired, two-way Student's $t$-test was performed using GraphPad Prism version 4.00 for Windows (GraphPad Software, San Diego, CA, USA). A $p$-value less than 0.05 was considered statistically significant.

\section{Results}

\section{Slit2/Robo mRNA Expression by Glioma Cells}

Slit2 has been shown to exhibit a repulsive effect for Robo-expressing axons of the commissural neurons, olfactory bulb neurons, and retinal ganglion cells through inactivation of a small GTPase, Cdc42. ${ }^{8}$ In human cancers, including gliomas, Slit2 is inactivated or downregulated. ${ }^{13-15}$ Two recent genomic studies using a large number of primary glioma specimens, including WHO grade II-IV tumors, validated the frequent downregulation of Slit2 expression in primary gliomas. ${ }^{26,27}$ Thus, we hypothesized that downregulation of Slit 2 in the invasive glioma cells suggests an inhibitory role of Slit2 in migration and invasion of glioma cells. To determine the role of Slit 2 in glioma cell migration and invasion, we first examined expression of Slit 2 and its receptor, Robo1, in NB tissues, primary glioma specimens, NHAs, and U373MG and SNB19 glioma cells. Because no reliable anti-Slit2 and anti-Robo1 antibodies were available, we isolated total RNA from snap-frozen NB tissues, four primary glioma specimens (WHO grade III, $\mathrm{J} 15, \mathrm{~J} 16$; and grade IV, J4, J5), NHA, and U373MG and SNB19 glioma cells and performed RT-PCR analyses to examine mRNA expression of Slit2 and Robo1 in these 
A.

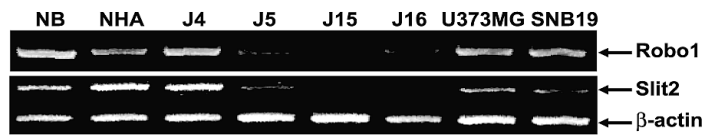

B.

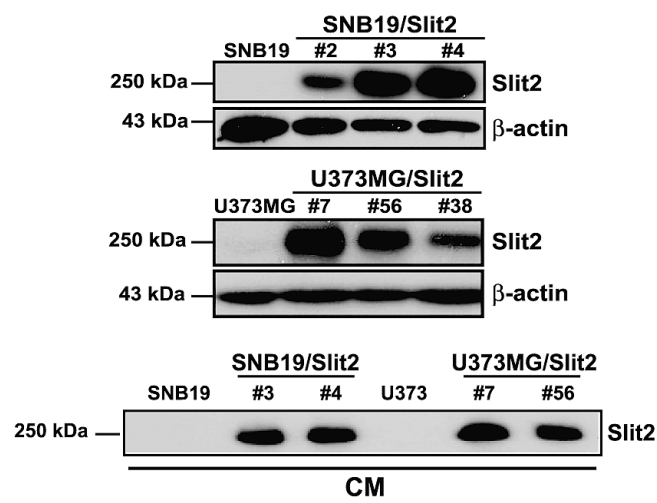

Fig. 1. Expression of Slit2 and Robo1 in primary glioma specimens and glioma cell lines. (A) Slit2 and Robo1 are expressed at lower levels in primary human glioma specimens and invasive SNB19 and U373MG glioma cells compared with normal human brain tissue (NB) and normal human astrocytes (NHA). Synthesized first-strand CDNA from total RNA of NB, primary glioma specimens (J4, J5, glioblastoma multiforme, WHO grade IV; J15 and J16, anaplastic oligodendroglioma, grade III), NHA, and SNB19 and U373MG glioma cells was used for PCR reactions. The length of synthesized CDNA fragments is 514 bp for Robo1 and 387 bp for Slit2, respectively. $\beta$-Actin was used as internal control. (B) Ectopic expression of Slit2 in SNB19 and U373MG glioma cells. Cell lysates (top two panels) or conditioned media (CM, bottom panel) from SNB19, SNB19 Slit2-expressing, U373MG, or U373MG Slit2-expressing cells were analyzed by immunoblot using an anti-c-Myc antibody. Lanes 2, 3, and 4 are SNB19 Slit2-expressing cell clones; lanes 7, 56 , and 38 are U373MG Slit2-expressing cell clones. $\beta$-Actin was used as a loading control. Results shown in $A$ and $B$ are representative of three independent experiments.

specimens and cells. As shown in Fig. 1A, expression of Slit2 was at lower levels in J5, J15, and J16 primary glioma specimens and in U373MG and SNB19 glioma cells compared with that in NB and NHA. Expression of Robo1 showed a similar pattern in tumor specimens but at similar levels among glioma cell lines. These results are consistent with their expression in primary glioma tissues and cell lines as previously reported. ${ }^{17,26,27}$

\section{Ectopic Expression of Slit2 Inbibits Cell Migration and Invasion of Glioma Cells In Vitro}

In human gliomas, Slit2 has been shown to be downregulated through hypermethylation at its promoter region in primary glioma specimens and cell lines, suggesting a suppressive role for Slit 2 during glioma progression. ${ }^{15,16,26,27}$ To determine the impact of Slit 2 expres- sion in glioma cell migration and invasion in vitro, SNB19 and U373MG cells were stably transfected with a human SL2myc cDNA containing a 6xc-Myc tag. ${ }^{17}$ Among various Slit2-expressing cell clones of SNB19 and U373MG cells, we chose three independent Slit2expressing cell clones of each cell line for our studies. As shown in the top panels of Fig. 1B, SNB19/Slit2 clone 3 and 4 cells expressed exogenous Slit 2 at high levels, while SNB19/Slit 2 clone 2 cells expressed Slit 2 at a lower level. In U373MG cells, U373MG/Slit2 clone 7 and 56 cells expressed Slit2 at high levels, and U373MG/Slit2 clone 38 cells expressed Slit 2 at a lower level. IB analyses of conditioned media (CM) of various glioma cells also showed that ectopically expressed Slit 2 was effectively secreted out of glioma cells at high levels, whereas no Slit2 was found in CM of parental glioma cells (Fig. 1B, bottom panel). Additionally, compared with the parental cells, the morphology of Slit2-expressing cell clones of SNB19 and U373MG cells was noticeably different, with the Slit 2 clones having a lesser ability to spread out on the culture dish (data not shown).

We determined whether the expression of Slit 2 affects cell proliferation and survival using WST-1 cell viability assays. ${ }^{20}$ As shown in Fig. 2A, in a 48-h culture, ectopic expression of Slit 2 only had minimal impact on cell growth and survival in U373MG and SNB19 cells. Next, we evaluated whether ectopically expressed Slit 2 affects migration of glioma cells by wound-healing assays. Serum-starved cells in six-well plates were scratched by a $200-\mu l$ pipette tip and cultured for an additional $24 \mathrm{~h}$ and $48 \mathrm{~h}$. As shown in Fig. 2B, at $24 \mathrm{~h} \mathrm{SNB19}$ Slit2-expressing cells encompassed approximately $5 \%$ of the scratched area compared with $30 \%$ by the parental cells (Fig. 2B). At 48 h, Slit2-expressing cells moderately migrated toward the center of the wound, whereas the parental cells completely closed the wound (Fig. 2B, lower panels).

We then tested whether expression of Slit 2 by glioma cells or treatment of glioma cells with recombinant Slit2 affects their ability to migrate through ECM proteincoated membranes using in vitro cell migration and invasion assays. An equal number of serum-starved glioma parental cells and their Slit2-expressing cell clones were separately placed into the top wells of a Boyden chamber. Media containing $0.5 \%$ FBS were placed to top and bottom wells of the chambers. In parallel, serum-starved parental SNB19 and U373MG cells were resuspended in CM from their Slit2-expressing cell clones and placed on the top wells on the Boyden chamber. The cells were then allowed to migrate through gelatin-coated membranes for $16 \mathrm{~h}$ (for SNB19 cells) or $8 \mathrm{~h}$ (for U373MG cells). As shown in Fig. 2C, U373MG and SNB19 Slit2expressing cell clones migrated through the membrane approximately 2 -fold less than their parental cells (left two panels). To a similar extent, treatment of parental SNB19 and U373MG cells with CM containing recombinant Slit2 also attenuated the capacity of these cells to migrate through the membrane (Fig. 2C, right two panels). To determine the effect of Slit2 expression or treatment of glioma cells with CM containing recombinant Slit2 on glioma cell invasion, we performed in 
A.

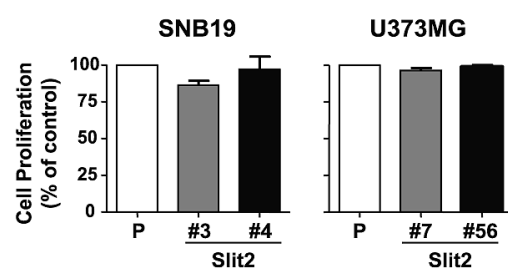

B.

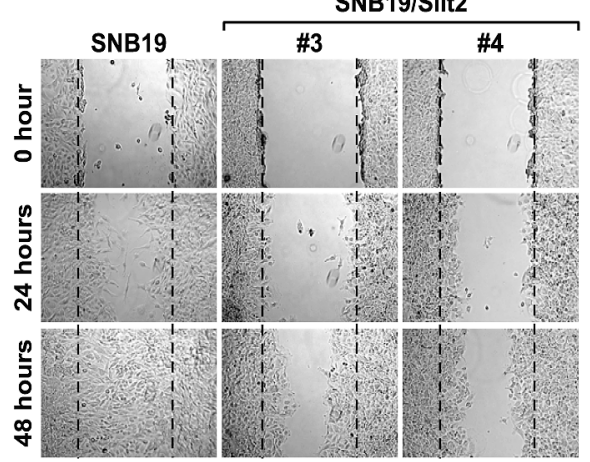

C.

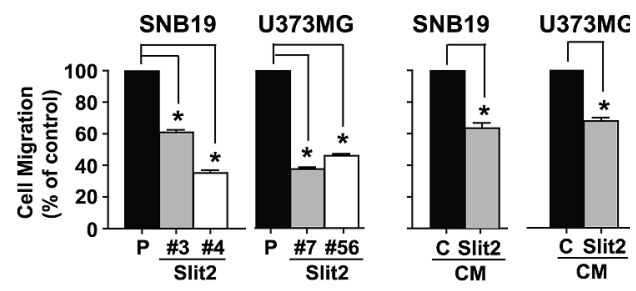

D.

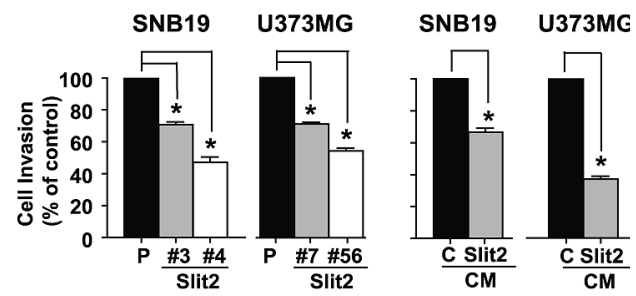

Fig. 2. Slit2 inhibits glioma cell migration and invasion in vitro. (A) Cell viability of parental (P) and Slit2-expressing cells was assessed by WST-1 assays in a 48-h culture in the absence of serum. Ectopic expression of Slit2 in SNB19 and U373MG cells has minimal impact on cell proliferation and survival. No significant difference was found. (B) Slit2 inhibits glioma cell migration in a wound-healing assay. Migration of parental SNB19 cells and U373MG cells (data not shown) or their Slit2-expressing cell clones was assessed in a wound closure at 0, 24, and $48 \mathrm{~h}$ after the wounds were generated. (C) Slit2 inhibits glioma cell migration in vitro. Slit2-expressing cell clones (left two panels) and their parental cells treated with Slit2-containing conditioned media ( $C M$; right two panels) have reduced ability to migrate through the gelatin-coated membranes. Abbreviations: P, parental; C, control. (D) Slit2 inhibits glioma cell invasion in vitro. Both Slit2-expressing cell clones (left two panels) and their parental cells treated with Slit2-containing CM (right two panels) have decreased ability to invade through membranes coated with growth factor reduced Matrigel. Cells migrating and invading through the membrane were counted in 10 random high-powered fields (total magnification, $\times 200$ ). Abbreviations: $P$, parental; $C$, control. For $A-D$, results represent three independent experiments performed in triplicate. Error bars in A, C, and D represent SD. ${ }^{*} p<0.05$, one-way analysis of variance followed by Newman-Keuls post hoc test.

vitro invasion assays for these glioma cells under similar conditions using a Growth Factor Reduced Matrigelcoated membrane. As shown in Fig. 2D, capacities of Slit2-expressing SNB19 and U373MG cell clones (left two panels) or parental SNB19 and U373MG cells treated with recombinant Slit2 (right two panels) to invade through the Matrigel-coated membrane were significantly impaired compared with that of their control cells. Taken together, these results showed that Slit2 expression inhibits glioma cell migration and invasion in vitro, consistent with its inhibitory role of cell migration as previously reported. ${ }^{11,12,14,17}$

\section{Expression of Slit2 by Glioma Cells Inhibits Tumor Cell Infiltration in the Brains of Animals}

Although Slit2 has been shown to inhibit cell motility in various types of human cells in vitro, whether expression of Slit 2 inhibits the invasion of cancer cells in their native tumor microenvironment in vivo has not been demonstrated. It is established that human glioma cells are intrinsically invasive in the brain. ${ }^{1,2}$ However, inva- sive behavior of glioma cells in vitro is often not correlated with their infiltrative phenotype in the brain. ${ }^{28}$ U373MG and SNB19 cells are able to form invasive tumors in the brain. In general, U373MG cells form invasive intracranial tumors in 60 days, ${ }^{29}$ while SNB19 cells developed small but invasive tumors in the brain within 3 weeks. ${ }^{30}$ To determine whether Slit 2 suppresses the invasion of glioma cells in the brain, we stereotactically implanted SNB19 parental cells and SNB19 Slit2expressing cell clones 3 and 4 into the brain of mice. As shown in Fig. 3A(a), 20 days after intracranial implantation, SNB19 parental cells formed small but invasive tumors in the brain. The SNB19 parental tumor has an irregular shape with single cells and cell clusters that invade along white matter tracts as well as into gray matter, perhaps also associated with blood vessels (Fig. 3A, a-c, arrows). In contrast, SNB19/Slit 2 clone 3 or clone 4 (data not shown) cells formed noninvasive ovalshaped tumors with a smoother border and necrosis in the center of tumor mass (Fig. 3A, e-g, arrows). To confirm that those single cells or cell clusters that are marked in the brain section in Fig. 3A $(\mathrm{a}-\mathrm{c})$ were SNB19 
A.

\section{SNB19}

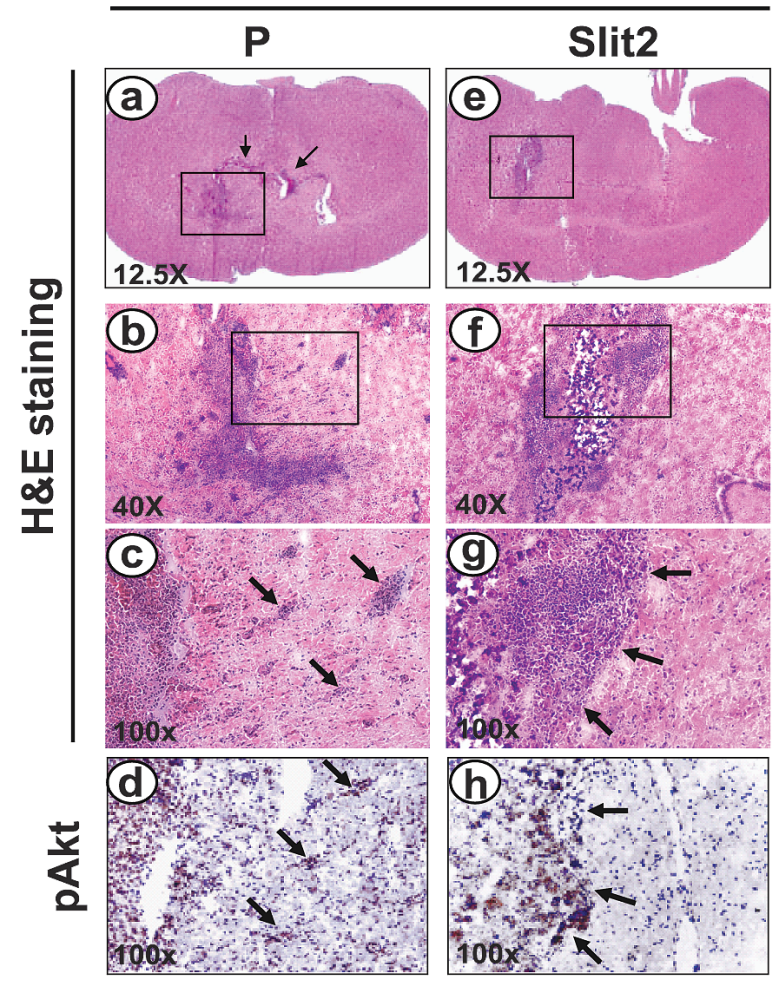

Fig. 3. Expression of Slit2 in SNB19 tumors inhibits glioma cell infiltration into the brain parenchyma of mice. (A) Representative results of hematoxylin and eosin ( $\mathrm{H} \& \mathrm{E} ; \mathrm{a}-\mathrm{C}, \mathrm{e}-\mathrm{g}$ ) and immunohistochemical staining using an anti-phosphorylated Akt (pAkt; $d$ and h) of glioma-bearing mouse brain cryosections. We stereotactically implanted $5 \times 10^{5}$ SNB19 parental $(P)$ or Slit2-expressing cells in a volume of $5 \mu$ phosphate-buffered saline into the brain. After 20 days, mice were euthanized and the brains were analyzed. $a-h$ represent a section of brain with an SNB19 parental tumor $(a-c)$ and a section of brain with an SNB19/Slit2 clone 3 tumor (Slit2; $\mathrm{e}-\mathrm{g}$ ); the enlarged regions marked in a and b are shown in b and c, respectively, and the enlarged regions marked in e and $f$ are shown in $f$ and $g$, respectively; $d$ is a sister section of $a-c$, and $h$ is a sister section of e-g. Arrows indicate the following: in a, the invasive parental tumor cells invading along white matter tracks, gray matter, and entering ventricle; in c, invasive tumor cells and disseminated tumor clusters away from the tumor mass; in $\mathrm{g}$, a smoother border of the SNB19 Slit2-expressing tumor; in d and h, pAktpositive tumor cells that appear to be an identical cell population, respectively, invaded brain parenchyma ( $b$ and $c$ ) or were noninvasive ( $f$ and $g$ ). Magnification: $a$ and $b, \times 12.5 ; b$ and $f, \times 40 ; c, d, g$, and $h, \times 100$. The intracranial glioma xenograft experiments were repeated in three independent studies along with two other SNB19 Slit2-expressing cell clones with similar results. (B) Expression of phosphorylated Akt (pAkt) in normal mouse brain, SNB19 parental $(P)$, and Slit2-expressing tumors. Cell lysates of normal mouse brain tissues and parental and Slit2-expressing SNB19 tumors were analyzed by immunoblot using an anti-pAkt antibody. $\beta$-Actin was used as a loading control. Results represent three independent experiments with similar results.

\section{Slit2 Expression Downregulates Cdc42 Activation through Its Receptor Robo1 in Glioma Cells}

Cdc42, a member of the small Rho GTPase family, plays important roles in cell migration by regulating the actin cytoskeleton. ${ }^{32,33}$ In the repulsive response by Slit2 stimulation, Robo1 receptor recruits srGAPs that inactivate Cdc42 GTPase activity, thus controlling neuron migration. ${ }^{8}$ To investigate whether Slit 2 expression in glioma cells regulates the activity of $\mathrm{Cdc} 42$, we tested the expression level of the GTP-bound form of endogenous Cdc42 in SNB19 and U373MG parental cells and Slit2-expressing cell clones. GTP-bound (active form) Cdc42 was separated from cell lysates of various parental glioma cells and Slit2-expressing cell clones with PAK-1 PBD agarose beads. The GTP-bound Cdc42 was examined using an anti-Cdc42 antibody. As shown in Fig. 4A, Slit2 expression in both SNB19 and U373MG cells significantly attenuated the GTP-binding activity of Cdc42 compared with their parental cells, whereas no alteration of $\mathrm{Cdc} 42$ protein expression was found. To validate the specificity of inhibition by recombinant Slit2 in CM, we performed immunodepletion with or without an anti-c-Myc antibody on the CM and assessed the impact of recombinant Slit2 on Cdc42 activity and cell migration. As shown in Fig. 4B, CM of SNB19/Slit2 clone 3 and U373MG/Slit 2 clone 56 containing recombinant Slit2 attenuated Cdc42 GTP-binding activities, 
A.

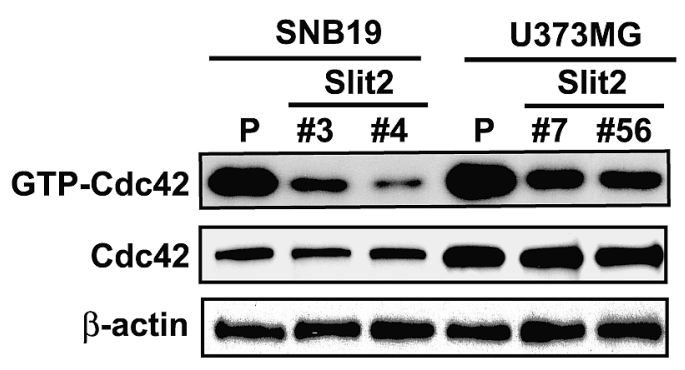

B.

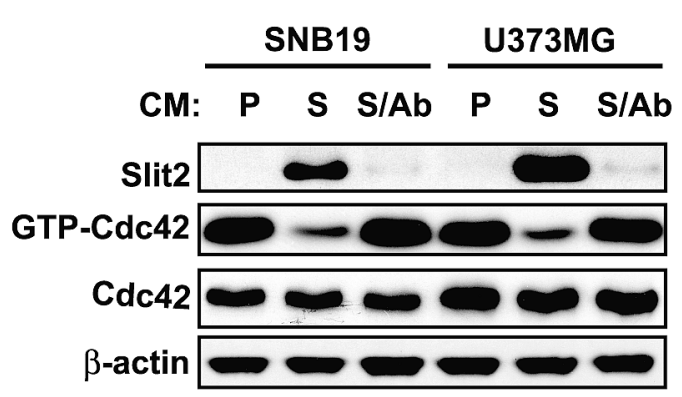

C.

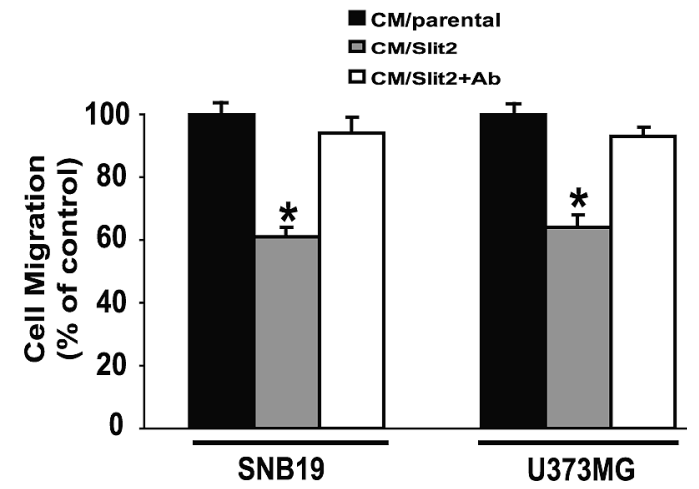

while removal of recombinant Slit 2 by immunodepletion prior to the Cdc 42 activity assays prevented Slit 2 inhibition. When the same batch of CM (parental CM, nondepleted CM-Slit2, or depleted-CM-Slit2) was applied to in vitro cell migration assays, recombinant Slit2 inhibited glioma cell migration while removal of Slit 2 from the CM by the antibody prior to the assays did not affect glioma cell migration (Fig. 4C). These results indicate that Slit2 regulates the activity of Cdc42 in glioma cells, consistent with previous reports showing Slit2 inhibition of Cdc42 function in several cell types. .11,12,14,17

Next, we assessed the impact of knockdown of Robo1, a cognate receptor for Slit2, in glioma cells by siRNA on Slit 2 inhibition of Cdc42 activity and cell migration. Because no reliable anti-Robo1 antibody was available for endogenous Robo1 detection in glioma cells, we used RT-PCR to validate siRNA knockdown of Robo1 in glioma cells. As shown in Fig. 5A, a pool of four separate siRNAs for Robo1 effectively depleted endogenous Robo1 in SNB19 and U373MG cells. When the Robo1 siRNA-transfected glioma cells were treated
Fig. 4. Slit2 specifically inhibits $\mathrm{Cdc} 42$ activity and glioma cell motility in vitro. (A and $B$ ) Slit2 expression (A) or treatment with recombinant Slit2 (B) attenuated GTPase activity of Cdc42 but not the expression of $\mathrm{Cdc} 42$ proteins in glioma cells. (A) Slit2 expression attenuated $\mathrm{Cdc} 42$ activity in glioma cells. Total cell lysates of parental (P) SNB19 and U373MG cells and various Slit2-expressing cell clones were analyzed for guanosine-5'-triphosphate (GTP) loading of Cdc42 using a Cdc42 activity assessment assay kit. (B) Recombinant Slit2 specifically inhibits $\mathrm{Cdc} 42$ activity. Parental cells of SNB19 and U373MG were treated with conditioned media (CM) collected from their parental cells (P) or Slit2-expressing cell clones (S). In parallel, CM of Slit2-expressing cell clones were incubated with $20 \mu \mathrm{g} / \mathrm{ml}$ of an anti-c-Myc antibody overnight at $4^{\circ} \mathrm{C}$ followed by immunodepletion of Slit2 from the CM prior to the assays (S/Ab). The assays were performed using CM of SNB19/Slit2 clone 3 and 4 cells and U373MG/Slit2 clone 7 and 56 cells with similar results. In both $A$ and $B$, the membranes were probed with antiCdc42 and anti- $\beta$-actin antibodies as loading controls. (C) In vitro cell migration. Treatment of parental SNB19 and U373MG cells with Slit2-containing $C M$ reduces ability to migrate through the gelatin-coated membranes, whereas removal of Slit2 from CM by immunodepletion prior to the assays prevents Slit2 inhibition of cell migration in vitro. Error bars in $C$ represent $S D{ }^{*} p<0.05$. For $A-C$, results represent three independent experiments with similar results.

with CM containing recombinant Slit2, Slit2 inhibition of Cdc42 GTP-binding activities was prevented compared with CM-treated cells that were transfected with control siRNA. When Robo1 knocked-down glioma cells were analyzed for their ability of migration through a precoated membrane in the Boyden chamber, depletion of Robo1 reversed Slit 2 inhibition of cell migration in the assays compared with control siRNA-transfected cells (Fig. 5C). Taken together, these data suggest that Slit2 specifically inhibits glioma cell motility via its receptor Robo1 through attenuating Cdc42 GTP binding activities in glioma cells.

\section{Slit2 Expression Does Not Affect the Expression of $\mathrm{N}$-Cadherin and $\boldsymbol{\beta}$-Catenin Proteins in Glioma Cells}

In the CNS, Slit2 exerts its repulsive function by decreasing N-cadherin-mediated cell adhesion. ${ }^{34}$ In mouse fibroblasts and human embryonic kidney $293 \mathrm{~T}$ cells, Slit2 inactivation of cell-cell adhesion is through Abl-mediated tyrosine phosphorylation of $\beta$-catenin, attenuating its affinity to $\mathrm{N}$-cadherin. The dissociated $\beta$-catenin translocates to the nucleus. Concurrently, dissociated $\mathrm{N}$-cadherin proteins were degraded. ${ }^{24,34}$ Thus, we determined the impact of Slit2 expression on tyrosine phosphorylation of $\beta$-catenin and association of $\mathrm{N}$-cadherin and $\beta$-catenin in glioma cells. After serum starvation, parental SNB19 cells and U373MG were treated with serum-free Slit2-containing CM or control CM for 10 $\min , 30 \mathrm{~min}, 3 \mathrm{~h}, 12 \mathrm{~h}$, and $24 \mathrm{~h}$. Then, tyrosine phosphorylation of $\beta$-catenin proteins was examined by IP and IB analyses. A basal level of tyrosine phosphorylation on $\beta$-catenin was found in both parental U373MG and SNB19 cells that were treated with control CM. 
A.

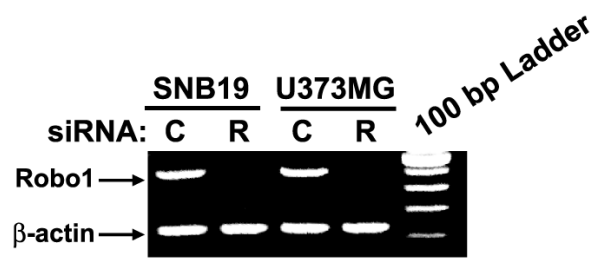

B.

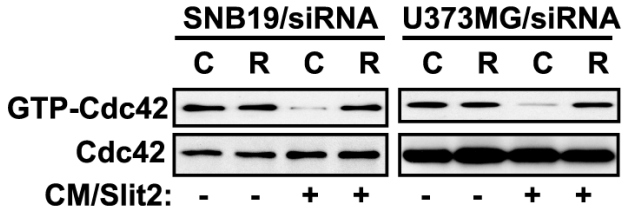

C.

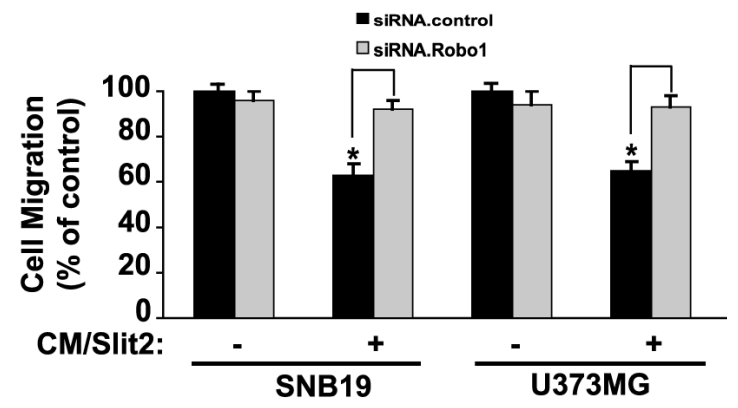

Fig. 5. Knockdown of Robo1 in glioma cells reverses Slit2 inhibition, restoring guanosine-5'-triphosphate (GTP) loading of Cdc42 and glioma cell motility. (A) Knockdown of Robo1 by small interfering RNA (siRNA) in glioma cells. Parental SNB19 and U373MG cells were transfected with a pool of four Robo1 siRNAs (R) and stealth control siRNA (C) for $48 \mathrm{~h}$. Total RNA prepared from the transfected SNB19 and U373MG cells was used for PCR reactions. The length of synthesized cDNA fragments for Robo1 is $514 \mathrm{bp}$. $\beta$-Actin was used as internal control. (B) Knockdown of Robo1 prevents Slit2 inhibition of Cdc42 activity. Total cell lysates of parental SNB19 and U373MG cells that were transfected with Robo1 siRNA (R) or control siRNA (C) followed by treatment with (+, Slit2containing conditioned media [CM]) or without (-, CM of parental cells) recombinant Slit2 were analyzed for GTP loading of Cdc42 using a Cdc42 activity assessment assay kit. The membranes were probed with an anti-Cdc42 antibody as a loading control. Knockdown of Robo1 by siRNA restored Cdc42 GTP loading activity in Slit2-treated glioma cells. (C) In vitro cell migration. Knockdown of Robo1 in parental SNB19 and U373MG cells with Robo1 siRNA but not control reverses the inhibition by recombinant Slit2 in CM of ability of glioma cells to migrate through the gelatin-coated membranes in vitro. Error bars in $C$ represent $S D{ }^{*} p<0.05$. For $A-C$, results represent three independent experiments with similar results.
However, no alteration of tyrosine phosphorylation on $\beta$-catenin was observed in glioma cells that were treated with Slit2-containing CM (data not shown). In parallel, parental and Slit2-expressing U373MG and SNB19 cells were analyzed by IP followed by IB using anti- $\beta$-catenin and anti-N-cadherin antibodies. As shown in Fig. 6A, $\mathrm{N}$-cadherin could be effectively coprecipitated with $\beta$-catenin in parental and Slit2-expressing U373MG and SNB19 cells, indicating that Slit2 did not affect the association of $\mathrm{N}$-cadherin and $\beta$-catenin in glioma cells. Next, we examined whether Slit 2 affects protein stability of $\mathrm{N}$-cadherin and $\beta$-catenin in $293 \mathrm{~T}$ and glioma cells. After a 24-h serum starvation, cell lysates of 293T, 293T Slit2-expressing cells, ${ }^{17}$ SNB19 parental and Slit2-expressing cells, and U373MG parental and Slit2-expressing cells were analyzed for expression of $\mathrm{N}$-cadherin and $\beta$-catenin. As shown in Fig. 6B, Slit2 expression in 293T cells resulted in significant decrease in levels of $\mathrm{N}$-cadherin and $\beta$-catenin proteins compared with parental 293T cells. However, in SNB19 and U373MG cells, Slit2 expression had no effect on the levels of $\mathrm{N}$-cadherin and $\beta$-catenin proteins. In parallel, parental U373MG and SNB19 cells were also incubated with Slit2-containing CM or control CM for 24 and 48 h. We found that treatment of recombinant Slit 2 did not cause any degradation of $\mathrm{N}$-cadherin and $\beta$-catenin proteins in these glioma cells (data not shown). Together, these results suggest that the inhibitory effects of Slit2 on cell migration and invasion of glioma cells did not involve modulation of phosphorylation $\beta$-catenin, association of $\mathrm{N}$-cadherin and $\beta$-catenin, or degradation of $\mathrm{N}$-cadherin and $\beta$-catenin proteins, thus differing from studies of Slit 2 modulation of $\mathrm{N}$-cadherin and $\beta$-catenin in fibroblasts and 293T cells. ${ }^{24,34}$

\section{Discussion}

In this study, we demonstrate for the first time that expression of a neuronal repulsive factor, Slit2, inhibits human glioma cell invasion into the brain parenchyma of mice. Slit2 is a chemorepulsive factor that controls axonal guidance and neuronal migration during development. ${ }^{4}$ Outside the CNS, in vitro studies have shown that Slit 2 attenuates cell migration of various types of human cells, including breast cancer and medulloblastoma cells. ${ }^{10-12,16,17}$ We identified Slit2 as a downregulated gene in DNA microarray analysis followed by realtime PCR of highly invasive human glioma U87MG Ang2-expressing cells versus noninvasive parental cells. ${ }^{19}$ Because the Slit2 gene is often silenced by hypermethylation at $\mathrm{CpG}$ islands in its promoter region in human cancers, including gliomas, ${ }^{13-15}$ we hypothesized that Slit2 expression impedes the insidious glioma cell infiltration in the brain in vivo. In the present study, we found that mRNA of Slit2 is expressed at lower levels in three of four primary glioma specimens and two highly invasive human glioma cell lines, SNB19 and U373MG, compared with NB and NHA. These data corroborate results of recent studies of Slit 2 expression in primary 
A.

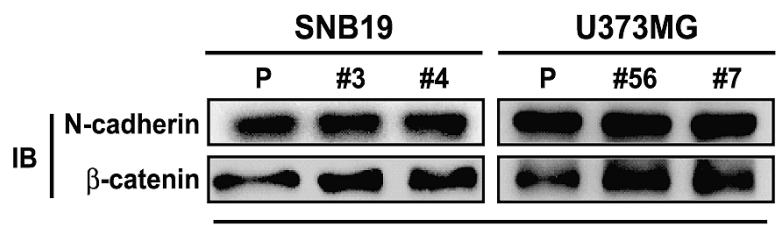

IP: N-cadherin

B.

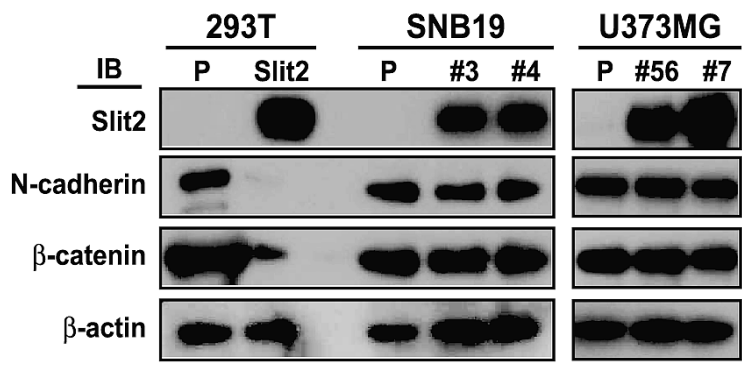

glioma specimens. In an analysis of 26 WHO grade II astrocytomas, 50 grade III oligodendrogliomas, and 77 grade glioblastoma multiforme clinical specimens, Slit2 expression was found to be downregulated compared with that in NB. ${ }^{26}$ Serial analysis of gene expression of 19 grade IV glioblastoma multiforme samples revealed that Slit2 is expressed at minimal levels. ${ }^{27}$ Due to lack of reliable antibodies against Slit2 and Robo1, we could only perform RT-PCR analyses to evaluate the impact of knockdown of endogenous Robo1 in U373MG and SNB19 cells. Moreover, knockdown of endogenous Robo1 in glioma cells prevented Slit2 inhibition of cell motility in vitro.

Our data also demonstrated that when highly migratory glioma cells were treated with Slit2, by either ectopic expression or recombinant protein using Slit2containing CM, the ability of these cells to migrate in wound-healing assays, migrate, and invade through precoated membrane was significantly attenuated. The inhibition by recombinant Slit 2 of cell migration was specific because immunodepletion of Slit 2 in CM diminished the inhibitory impact on cell motility by Slit2-containing CM. When invasive SNB19 cells that express exogenous Slit 2 were implanted into the brain of mice, SNB19 glioma cell infiltration into the surrounding brain parenchyma of mice was markedly impaired. In addition, we found that Slit 2 treatment did not influence glioma cell proliferation and survival in vitro and in vivo (data not shown). These results provide convincing evidence that the neuronal repulsive factor Slit2 exerts an inhibitory role in glioma cell invasion in vivo. These data corroborate previous in vitro studies that Slit 2 inhibits cell migration and invasion of various types of mammalian cells. ${ }^{10-12,16,17}$

The inhibitory effects of Slit2-Robo interaction on migration and invasion of various cell types are medi-
Fig. 6. Slit2 expression did not affect the expression of $\mathrm{N}$-cadherin and $\beta$-catenin in glioma cells. (A) Immunoprecipitation (IP) and immunoblot (IB) analyses. Cell lysates of parental (P) and Slit2expressing cell clones of SNB19 and U373MG cells were subjected to IP using an anti- $\mathrm{N}$-cadherin antibody followed by IB using anti- $\mathrm{N}$-cadherin and anti- $\beta$-catenin antibodies. $\beta$-Catenin was effectively precipitated with $\mathrm{N}$-cadherin in both parental and Slit2expressing cells. (B) IB analyses. Human embryonic kidney 293T and human glioma SNB19 and U373MG parent cells (P) and their Slit2-expressing cell clones were analyzed for expression of Slit2, $\mathrm{N}$-cadherin, and $\beta$-catenin by IB using anti-c-Myc, anti- $\mathrm{N}$-cadherin, and anti- $\beta$-catenin antibodies. The molecular weights of proteins are, for Slit2 with c-Myc tags, $250 \mathrm{kDa}$; N-cadherin, $136 \mathrm{kDa}$; and $\beta$-catenin, $92 \mathrm{kDa}$. $\beta$-Actin was used as a loading control. Slit2 expression significantly decreased expression of $\mathrm{N}$-cadherin and $\beta$-catenin in 293T cells but did not affect the expression of these proteins in SNB19 and U373MG glioma cells. For A and B, results represent three independent experiments with similar results.

ated by several downstream effectors. In leukocytes and breast cancer cells, Slit2 inhibits chemokine/CXCR4mediated cell migration through inhibition of ERK1/2 activation. ${ }^{10,16}$ In CNS and human meduloblastoma cells, Slit 2 controls cell motility by attenuating activity of the small Rho GTPase Cdc42. ${ }^{8,17}$ In vascular smooth muscle cells, the proteolytic fragment of the $\mathrm{N}$-terminal region of Slit 2 peptide that exhibits distinct cell association characteristics ${ }^{35}$ inhibits cell migration by impeding platelet-derived growth factor-activated Rac1. ${ }^{12}$ In this study, we found that Slit2 expression or treatment of glioma cells with CM containing recombinant Slit2 significantly inhibited Cdc 42 but not Rac1 activity (data not shown). Slit2 inhibition of cell migration and Cdc42 activity was specific because immunodepletion of recombinant Slit2 from CM prior to the assays significantly attenuated Slit 2 inhibition. When the cognate receptor of Slit2, Robo1, was knocked down by siRNA, Slit2 inhibition of Cdc42 activity and cell motility was reversed. Additionally, Slit2 inhibition of U373MG and SNB19 cell motility is not in agreement with previous results demonstrating that exogenous Slit2 did not alter U251MG cell invasion behavior in vitro. ${ }^{17} \mathrm{~A}$ plausible explanation for this inconsistency is that expression of regulators of small Rho GTPases, such as guanine exchange factors (GEFs) that activate Cdc42 and Rac1, differs in various glioma cells. For example, a bipartite GEF, ELMO1 and Dock180, is highly expressed in U373MG and SNB19 cells but expressed at low levels in U251MG and several other glioma cell lines. ${ }^{22}$ Because this bipartite GEF is highly expressed by actively invading glioma cells in the invasive regions of primary glioma specimens, ${ }^{22}$ the lack of modulators of Cdc 42 in U251MG cells probably attributes to the unresponsiveness to Slit2.

Recent reports proposed other mechanisms by which Slit2-Robo interaction modulates cell migration. In 
chick neural retinal cells and mouse fibroblasts, Slit2 induces formation of a Robo/Abl/Cable-N-cadherin/ $\beta$-catenin complex and tyrosine phosphorylation of $\beta$-catenin that moves into nucleus, resulting in disassociation of $\beta$-catenin with $\mathrm{N}$-cadherin, and degradation of $\beta$-catenin and $\mathrm{N}$-cadherin proteins. ${ }^{24,34}$ Alternatively, loss of $\mathrm{N}$-cadherin function could also be caused by proteolytic cleavage releasing $\mathrm{N}$ - and $\mathrm{C}$-terminal peptides of $\mathrm{N}$-cadherin. ${ }^{36}$ Because expression of $\mathrm{N}$-cadherin correlates with high-grade glioma progression, ${ }^{37}$ we examined whether Slit 2 treatment affected expression and stability of $\mathrm{N}$-cadherin and $\beta$-catenin proteins in invasive glioma cells. Our data show that Slit2 expression had no impact on protein stability in glioma cells, whereas a significant decrease in levels of expression of $\beta$-catenin and $\mathrm{N}$-cadherin was evident in Slit2-expressing $293 \mathrm{~T}$ cells. We also did not observe any changes in $\beta$-catenin phosphorylation and association of $\beta$-catenin and $\mathrm{N}$-cadherin in Slit2-treated glioma cells. In addition, no alteration in the cleavage of $\mathrm{N}$-cadherin in Slit2-treated versus nontreated glioma cells was found. Clearly, our findings in human glioma cells are different from previous studies in fibroblasts and human embryonic kidney 293T cells in that Slit 2 affects expression and stabilities of $\mathrm{N}$-cadherin and $\beta$-catenin proteins in these cells. Taken together, the signaling of Slit2-Robo axis in inhibiting cell invasion appears to be complex in glioma cells and distinct from its action in neural cells, fibroblasts, and human breast cancer cells, thus warranting further investigation.

In summary, this study demonstrates an inhibitory role of Slit2 in human glioma cell migration in vitro and tumor cell infiltration into the brain parenchyma in vivo. Our results show that Slit2 impedes glioma cell invasion through inhibition of Rho GTPase activity of Cdc42 without affecting the expression of $\mathrm{N}$-cadherin and $\beta$-catenin in invasive glioma cells. Because diffuse invasion by single glioma cells into the NB is a major obstacle in treatment of patients with malignant gliomas, understanding how neurorepulsive cues such as the Slit-Robo axis modulate glioma cell motility is highly significant and could provide insight for development of effective treatments for these deadly brain cancers.

\section{Acknowledgments}

We thank N. Balass for proofreading the manuscript. This article was supported by grants from the National Institutes of Health (CA102011 and CA130966). J.Y.W. also received grants from the National Institutes of Health (CA114197, CA107193, and DA0176404); S.-Y.C. received support from the American Cancer Society (grant RSG CSM-107144); and S.-Y.C. and B.H. received support from the Hillman Foundation (Innovative Research Scholar Award).

\section{References}

1. Kleihues P, Cavenee WK. Pathology and Genetics: Tumours of the Nervous System. Lyon, France: IARC Press, 2000.

2. Furnari FB, Fenton T, Bachoo RM, et al. Malignant astrocytic glioma: genetics, biology, and paths to treatment. Genes Dev. 2007;21:26832710.

3. Giese A, Bjerkvig R, Berens ME, Westphal M. Cost of migration: invasion of malignant gliomas and implications for treatment. J Clin Oncol. 2003;21:1624-1636.

4. Wong K, Park HT, Wu JY, Rao Y. Slit proteins: molecular guidance cues for cells ranging from neurons to leukocytes. Curr Opin Genet Dev. 2002;12:583-591.

5. Dickson BJ, Gilestro GF. Regulation of commissural axon pathfinding by slit and its Robo receptors. Annu Rev Cell Dev Biol. 2006;22:651675.

6. Wu W, Wong K, Chen J, et al. Directional guidance of neuronal migration in the olfactory system by the protein Slit. Nature. 1999;400:331336.

7. De Bellard ME, Rao Y, Bronner-Fraser M. Dual function of Slit2 in repulsion and enhanced migration of trunk, but not vagal, neural crest cells. J Cell Biol. 2003;162:269-279.

8. Wong $K$, Ren $X R$, Huang $Y Z$, et al. Signal transduction in neuronal migration: roles of GTPase activating proteins and the small GTPase Cdc42 in the Slit-Robo pathway. Cell. 2001;107:209-221.

9. Etienne-Manneville S, Hall A. Rho GTPases in cell biology. Nature. 2002;420:629-635.

10. Wu JY, Feng L, Park HT, et al. The neuronal repellent Slit inhibits leu- kocyte chemotaxis induced by chemotactic factors. Nature. 2001; 410:948-952.

11. Guan $H, Z u$ G, Xie $Y$, et al. Neuronal repellent Slit2 inhibits dendritic cell migration and the development of immune responses. J Immunol. 2003;171:6519-6526.

12. Liu D, Hou J, Hu X, et al. Neuronal chemorepellent Slit2 inhibits vascular smooth muscle cell migration by suppressing small GTPase Rac1 activation. Circ Res. 2006;98:480-489.

13. Dallol A, Da Silva NF, Viacava P, et al. SLIT2, a human homologue of the Drosophila Slit2 gene, has tumor suppressor activity and is frequently inactivated in lung and breast cancers. Cancer Res. 2002; 62:5874-5880.

14. Dallol A, Krex D, Hesson $L$, et al. Frequent epigenetic inactivation of the SLIT2 gene in gliomas. Oncogene. 2003;22:4611-4616.

15. Mertsch S, Schmitz N, Jeibmann A, et al. Slit2 involvement in glioma cell migration is mediated by Robo1 receptor. J Neurooncol. 2008;87:1-7.

16. Prasad A, Fernandis AZ, Rao Y, Ganju RK. Slit protein-mediated inhibition of CXCR4-induced chemotactic and chemoinvasive signaling pathways in breast cancer cells. J Biol Chem. 2004;279:9115-9124.

17. Werbowetski-Ogilvie TE, Seyed Sadr M, Jabado N, et al. Inhibition of medulloblastoma cell invasion by Slit. Oncogene. 2006;25:51035112.

18. Furnari FB, Lin H, Huang HS, Cavenee WK. Growth suppression of glioma cells by PTEN requires a functional phosphatase catalytic domain. Proc Natl Acad Sci U S A. 1997;94:12479-12484. 
19. Hu B, Guo P, Fang $Q$, et al. Angiopoietin-2 induces human glioma invasion through the activation of matrix metalloprotease-2. Proc Natl Acad Sci U S A. 2003;100:8904-8909.

20. Huang PH, Mukasa A, Bonavia R, et al. Quantitative analysis of EGFRvIII cellular signaling networks reveals a combinatorial therapeutic strategy for glioblastoma. Proc Natl Acad Sci U S A. 2007;104:1286712872.

21. Tao H, Berno AJ, Cox DR, Frazer KA. In vitro human keratinocyte migration rates are associated with SNPs in the KRT1 interval. PLoS ONE. 2007;2:e697.

22. Jarzynka MJ, Hu B, Hui KM, et al. ELMO1 and Dock180, a bipartite Rac1 guanine nucleotide exchange factor, promote human glioma cell invasion. Cancer Res. 2007;67:7203-7211.

23. Hu B, Jarzynka MJ, Guo P, et al. Angiopoietin 2 induces glioma cell invasion by stimulating matrix metalloprotease 2 expression through the alphavbeta1 integrin and focal adhesion kinase signaling pathway. Cancer Res. 2006;66:775-783.

24. Rhee J, Buchan T, Zukerberg L, Lilien J, Balsamo J. Cables links Robobound $\mathrm{Abl}$ kinase to $\mathrm{N}$-cadherin-bound beta-catenin to mediate Slit-induced modulation of adhesion and transcription. Nat Cell Biol. 2007;9:883-892.

25. Imanishi Y, Hu B, Jarzynka MJ, et al. Angiopoietin-2 stimulates breast cancer metastasis through the alpha(5)beta(1) integrin-mediated pathway. Cancer Res. 2007;67:4254-4263.

26. Sun L, Hui AM, Su Q, et al. Neuronal and glioma-derived stem cell factor induces angiogenesis within the brain. Cancer Cell. 2006;9:287300.

27. Parsons DW, Jones $S$, Zhang $X$, et al. An integrated genomic analysis of human glioblastoma multiforme. Science. 2008;321:1807-1812.

28. Bellail AC, Hunter SB, Brat DJ, Tan C, Van Meir EG. Microregional extracellular matrix heterogeneity in brain modulates glioma cell invasion. Int J Biochem Cell Biol. 2004;36:1046-1069.

29. Branle F, Lefranc F, Camby I, et al. Evaluation of the efficiency of chemotherapy in in vivo orthotopic models of human glioma cells with and without $1 \mathrm{p} 19 \mathrm{q}$ deletions and in C6 rat orthotopic allografts serving for the evaluation of surgery combined with chemotherapy. Cancer. 2002;95:641-655.

30. Kondraganti S, Mohanam S, Chintala SK, et al. Selective suppression of matrix metalloproteinase- 9 in human glioblastoma cells by antisense gene transfer impairs glioblastoma cell invasion. Cancer Res. 2000;60:6851-6855.

31. Li Y, Guessous F, Kwon S, et al. PTEN has tumor-promoting properties in the setting of gain-of-function p53 mutations. Cancer Res. 2008;68:1723-1731.

32. Hall A. Rho GTPases and the actin cytoskeleton. Science. 1998;279: 509-514.

33. Machesky LM, Insall RH. Signaling to actin dynamics. J Cell Biol. 1999;146:267-272.

34. Rhee J, Mahfooz NS, Arregui C, et al. Activation of the repulsive receptor Roundabout inhibits $\mathrm{N}$-cadherin-mediated cell adhesion. Nat Cell Biol. 2002;4:798-805

35. Brose $\mathrm{K}$, Bland KS, Wang KH, et al. Slit proteins bind Robo receptors and have an evolutionarily conserved role in repulsive axon guidance. Cell. 1999:96:795-806.

36. Uemura K, Kuzuya A, Aoyagi N, et al. Amyloid beta inhibits ectodomain shedding of $\mathrm{N}$-cadherin via down-regulation of cell-surface NMDA receptor. Neuroscience. 2007;145:5-10.

37. Asano K, Duntsch CD, Zhou Q, et al. Correlation of N-cadherin expression in high grade gliomas with tissue invasion. J Neurooncol. 2004;70:3-15. 